\begin{tabular}{|l|}
\hline Marine Biology \\
\hline C Springer-Verlag 2001 \\
\hline DOI 10.1007/s00227-001-0710-8 \\
\hline
\end{tabular}

\title{
Average nanorough skin surface of the pilot whale (Globicephala melas, Delphinidae): considerations on the self-cleaning abilities based on nanoroughness
}

C. Baum $^{1,2}$, W. Meyer ${ }^{2,}$, R. Stelzer ${ }^{3}$, L.-G. Fleischer ${ }^{4}$ and D. Siebers ${ }^{1}$

(1) Stiftung Alfred-Wegener-Institut für Polar- und Meeresforschung, Am Handelshafen 12, 27570 Bremerhaven, Germany

(2) Anatomisches Institut, Tierärztliche Hochschule Hannover, Bischofsholer Damm 15, 30173 Hannover, Germany

(3) Institut für Tierökologie und Zellbiologie, Tierärztliche Hochschule Hannover, Bünteweg 17d, 30559 Hannover, Germany

(4) Institut für Lebensmitteltechnologie, Biotechnologie-Zentrum, Technische Universität Berlin, Amrumer Strasse 32, 13353 Berlin, Germany

E-mail: wilfried.meyer@tiho-hannover.de

Phone: +49-511-5867215

Fax: +49-511-5867232

Received: 29 May 2001 / Accepted: 27 August 2001 / Published online: 14 November 2001

Communicated by O. Kinne, Oldendorf/Luhe

\begin{abstract}
In aquatic environments, the biofouling process is assumed to initiate from the conditioning layer of absorbed organic carbon residues on wetted surfaces. Microfouling organisms attach to this conditioning layer, building up a biofilm on which further biofouling proceeds. In dolphins, biofouling reduces hydrodynamic efficiency and may negatively affect health if not managed. In the present study we examined the skin surface of the pilot whale (Globicephala melas). Employing cryo-scanning electron microscopic techniques combined with various sample preparations, the skin displayed an average nanorough surface characterized by a pattern of nanoridge-enclosed pores; the average pore size (approximately $0.20 \mu \mathrm{m}^{2}$ ) was below the size of most marine biofouling organisms. Further, the implications of this type of surface to the self-cleaning abilities of the skin of pilot whales are discussed, based on reduced available space for biofouler attachment, the lack of any particular microniches as shelters for biofoulers, and the challenges of turbulent water flow and liquid-air interfaces during surfacing and jumping of the dolphin.
\end{abstract}

\section{Introduction}

In the marine environment, adhesive polysaccharides within the pool of dissolved organic matter (DOM) can form colloids and gel particles spontaneously (Chin et al. 1998; Passow 2000). This process depends on the concentration and absorptive properties of the saccharide precursors, mainly consisting of sulfated acyl-heteropolysaccharides (Aluwihare et al.1997; Aluwihare and Repeta 1999). 
The transformation of small dissolved molecules $(\leq 0.2 \mu \mathrm{m})$ into sinking particles $(\geq 0.2 \mu \mathrm{m})$ is considered essential to the permanent cycling of marine organic matter (Passow 2000). Since the differentiation between dissolved and particulate organic matter originates from the usual filtration of sea water, bacteria and diatoms $(\geq 0.2 \mu \mathrm{m})$ exudating heteropolysaccharides belong to the particulate

fraction.

Absorbed by the charged and hydrophobic surfaces of organisms or underwater structures, adhesive organic matter forms a conditioning organic layer. Microfouling organisms attaching to and growing on this layer initiate the formation of a mucilaginous and mainly polysaccharide-based biofilm (Wahl 1989; Cooksey and Wigglesworth-Cooksey 1995; Beveridge et al. 1997; Stoodley et al. 1999). Biofilms modified for adhesion, nutrition or defense by microfouling organisms may reduce hydrodynamic efficiency and create health problems for the aquatic plants or animals they cover.

However, taking advantage of cryo-scanning electron microscopic (C-SEM) techniques in combination with cryo-conservation, we were able to show that the skin surface of the pilot whale (Globicephala melas) exhibits remarkably low concentrations of epibiontic organisms and salt crystals. Most surprisingly, freeze-etching resulted in the partition of the skin surface into hydrophilic non-lipidic and hydrophobic lipidic sectors. These sectors varied in thickness and diameter (about 1-5 $\mu \mathrm{m}$ and $10-100 \mu \mathrm{m}$, respectively), indicating the presence of a skin-covering fluid; this is patchy and may be derived from the dolphin skin (Baum et al. 2000, 2001a, $\underline{b}$ ). In addition, at higher magnifications of this skin surface, plane, small tips could be demonstrated; these may evidence desmosomal junctions in the outermost stratum corneum cells.

The present study is aimed at establishing whether a biological structure is related to the morphology described above. In this connection, trypsin was applied to the skin surface of the pilot whale in order to digest non-desmosomal material. We compared the surface structure of the trypsinated skin samples to fresh-frozen, formol- and Bouin-fixed samples. The specific skin structure was also compared to the size classes of marine biofoulers, and options of attachment-detachment mechanisms during surfacing or jumping of whales are considered.

\section{Materials and methods}

A total of 50 skin samples from 30 pilot whales (Globicephala melas) obtained from the Faroe Islands in 1999 were cut into blocks $(5 \times 5 \times 5 \mathrm{~mm})$. These fresh blocks were used unfixed, but were stored and

viewed deep frozen (liquid nitrogen at $-196^{\circ} \mathrm{C}$ ), in order to preserve the natural character of the samples. Alternatively, they were fixed in $4 \%$ formol for further enzymatic digestion or in Bouin's fixative, whereby the latter is recommended for the stabilization of glycoconjugates (Meyer 1986). After formol fixation, a batch of the blocks was rinsed in PBS (phosphate-buffered saline, $\mathrm{pH}$ 7.4) for $12 \mathrm{~h}$, and then incubated with $0.25 \%$ trypsin (10,000 units, Sigma) in PBS (pH 7.4) for 2, 6 and $12 \mathrm{~h}$ at $4^{\circ} \mathrm{C}$. The blocks were placed onto copper sampler holders and then stored deep frozen in liquid nitrogen $\left(-196^{\circ} \mathrm{C}\right)$. Aliquots of the blocks were also formol fixed and dehydrated in ethanol and dried chemically using tetramethylsilane (Dey 1989). For structural comparison, we used cellulose-acetate filters (Sartorius, SM 11307, size exclusion $0.22 \mu \mathrm{m}$ ). C-SEM photographs were taken after freezing, after freeze-etching and freeze-drying of the samples under optical control in the cryo-electron microscope ETEC autoscan (for details, see Baum et al. 2000). In brief, samples were viewed under high vacuum conditions $\left(1.3 \times 10^{-3} \mathrm{~Pa}\right)$ and low temperature $\left(-196^{\circ} \mathrm{C}\right)$, with an accelerating voltage of

the electron beam of $10 \mathrm{kV}$ and an emission current of $175 \mu \mathrm{A}$. In order to determine the areas of the nanoridge-enclosed pores, size-calibrated images of the skin samples were converted to a monochrome gray level by erosion and contrasting routines with the morphometric software Analysis 2.1 (Soft

Imaging System). The pore sizes measured were sorted into $0.1 \mu \mathrm{m}^{2}$ classes. 


\section{Results}

Taking advantage of C-SEM techniques in combination with cryo-conservation, conventional prefixation and dehydration (or additional coating) of the sample was not necessary, and thus the dislocation of biofoulers and chemical denaturation of the samples was avoided. In agreement with previous studies, the freshly frozen skin samples of pilot whale collected in 1999 exhibited low concentrations of epibionts, similar to the freshly frozen samples from 1997 (Baum et al. 2000). The skin surface displayed small tips arising from the average smooth background (Fig. 1 ), no pores were present. Employing mild-freeze etching (sample dehydration developed along the gradient of the water content), material between the tips dehydrated, leaving behind the pattern of pores enclosed by an entangled network of nanoridges, which were recovered as the underlying part of the tips (not shown). Since nanoridges are known from aldehyde-fixed samples as the derivatives of the desmosomal junctions of the outermost corneocytes (e.g. Pfeiffer and Jones 1993), we applied an enzymatic hydrolysis, commonly used to enhance epitope presentation in immunodetection of (desmosomal) keratins of formol-fixed skin samples. The trypsin digestion revealed the presence of a relief of nanoridges (approximately $100 \mathrm{~nm}$ deep) on both sides of the cell surface of the corneocytes (approximately $300 \mathrm{~nm}$ thick) (Fig. 2). It was remarkable that after the digestion procedure, in contrast to the non-digested aldehyde-fixed samples, the corneocytes (ca. $30 \times 50 \mu \mathrm{m}$ ) were often seen partly detached from the underlying cells, not as single scales, but in larger assemblies (ca. $100 \times 300 \mu \mathrm{m}$ ).

Nanoridges enclosing pores were demonstrated after trypsin digestion comparable to the above-mentioned structure in the freshly freeze-etched skin samples and Bouin-fixed samples (Fig. 3 ). The size of the nanoridges was only randomly affected by the digestion even under prolonged proteolysis (2-24 h). As with the freshly frozen, non-etched samples, the nanoridge-enclosed pores were partly obscured by a medium filling the pores of fixed skin samples (Bouin's fixative and formol). In comparison to the average nanorough surface of the cellulose-acetate filter membranes (pore size exclusion $0.22 \mu \mathrm{m}$, Fig. 4) prepared from nanoparticles, the surface structure of the corneocytes displayed a greater degree of smoothness and structural integrity (cf. Figs. $\underline{3}$ and $\underline{4}$ ). 

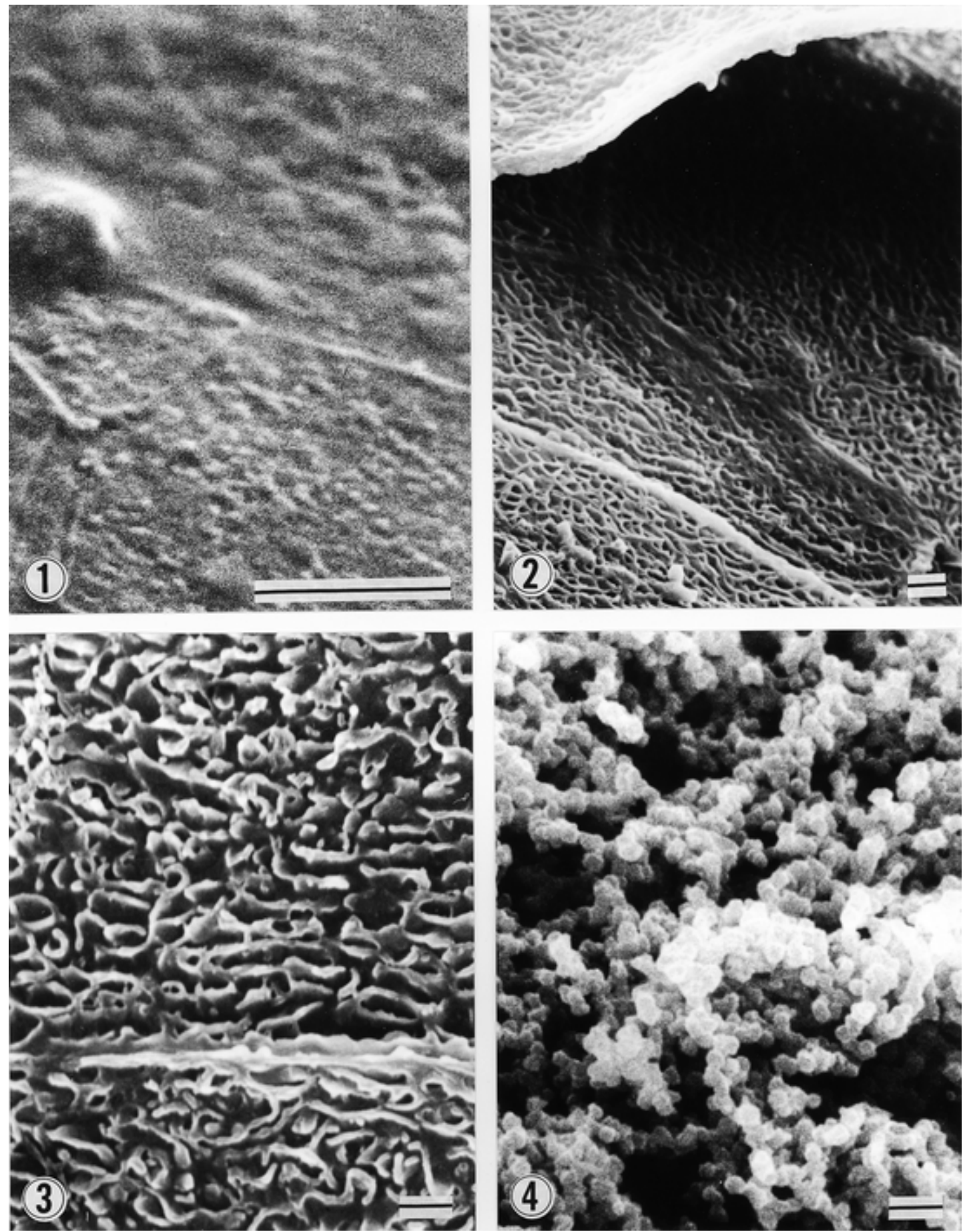

Fig. 1. Globicephala melas. Cryo-scanning electron microscopic images of the skin surface (non-fixed) of pilot whale displayed an even, smooth surface. Only the small tips of the nanoridges are seen compared to the fixed sample in Figs. 2 and 3 . The method used ensured that the medium smoothing the surface can be identified. Scale bar: $10 \mu \mathrm{m}$

Fig. 2. Globicephala melas. After trypsin hydrolysis of formol-fixed samples an entangled network of nanoridges was visible showing the derivatives of the desmosomal junctions of the corneocytes. These nanoridges enclosed small pores. It is remarkable that after the digestion procedures the desquamation was enhanced. Scale bar: $1 \mu \mathrm{m}$

Fig. 3. Globicephala melas. Non-digested skin samples (fixed in Bouin's fixative), the pores enclosed by the nanoridges are displayed very clearly.Scale bar: $1 \mu \mathrm{m}$

Fig. 4. Cellulose-acetate membrane filter (Sartorius, SM 11307, pore size exclusion $0.20 \mu \mathrm{m}$ ) made by 
nanoparticles exhibited a higher degree of roughness than the surface of the corneocytes of the pilot whale. Scale bar: $1 \mu \mathrm{m}$

Using C-SEM techniques and conventional SEM preparation methods, we determined the amount of shrinkage of the skin samples usually observed after fixation and chemical dehydration. Estimated from the size of non-dehydrated cryo-fixed samples, all other preparation methods reported above induced isometric size reduction of about 10-15\% (freeze-etching, fixation using Bouin's fixative and formol in combination with cryo-scanning microscopy, and also fixation in formol followed by chemical dehydration in ethanol and tetramethysilane). We therefore recalculated the pore sizes analyzed for the freshly freeze-etched and the aldehyde-fixed samples within a $10 \%$ variation. The pores measured occupied about $20 \%$ of the plane surface area. In general, the pore sizes ranged between 0.1 and $1.2 \mu \mathrm{m}^{2}$ and were dominated by the 0.10 and $0.20 \mu \mathrm{m}^{2}$ fractions (approximately $90 \%$ of all pore sizes measured) (Fig. $\underline{5}$ ).

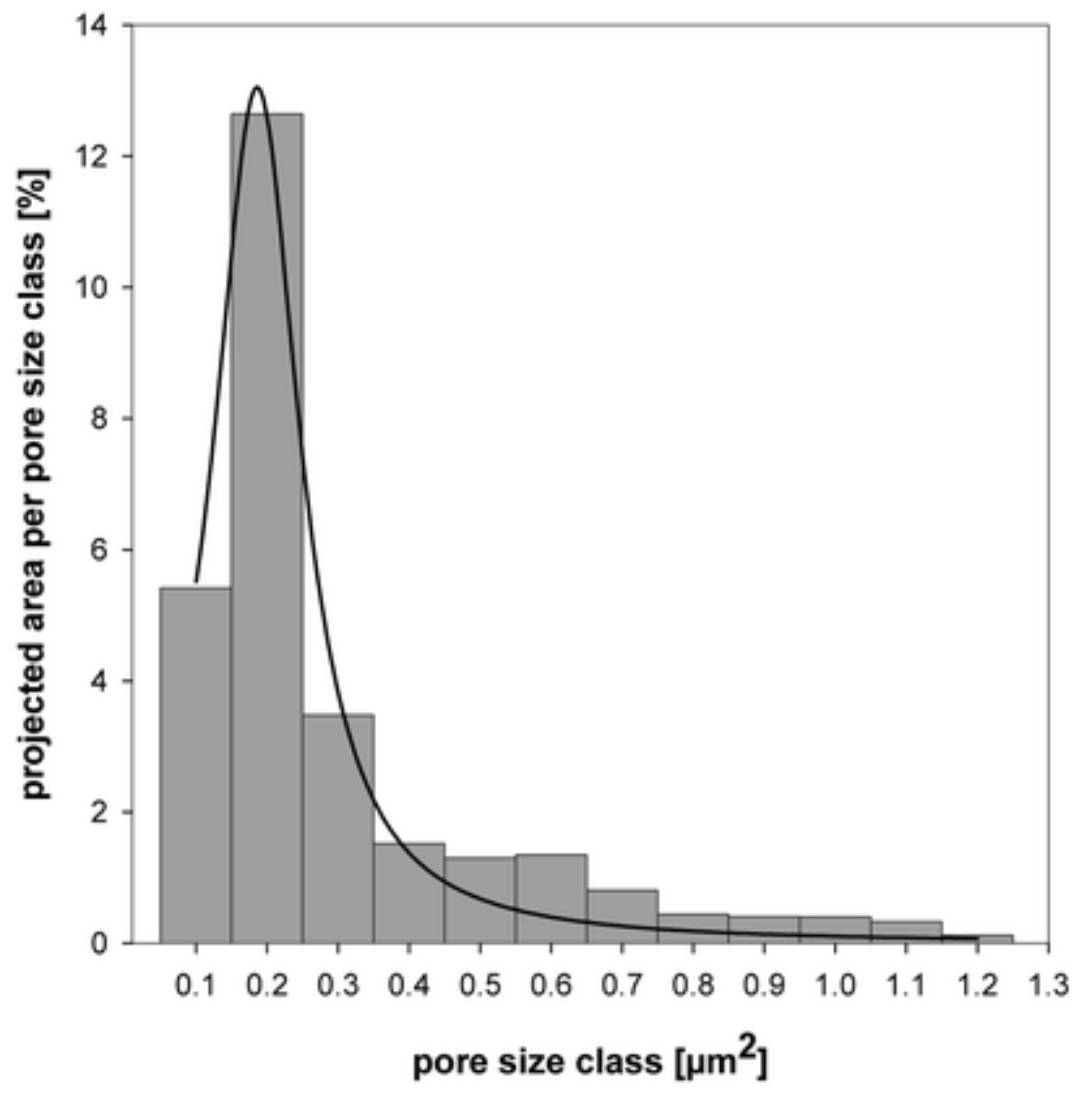

Fig. 5. Globicephala melas. Pore size distribution in the corneocytes of the pilot whale. Approximately $20 \%$ of the corneocyte surface was covered by pores. The distribution is dominated (nearly $50 \%$ ) by the $0.20 \mu \mathrm{m}^{2}$ fraction. Data fitted to the Lorentzian density distribution $\left(R=0.99283, R^{2}=0.9857193\right.$, standard error of estimate $=0.4748$ )

\section{Discussion and conclusions}

It was obvious from the results of our previous C-SEM study (Baum et al.2000), that the skin surface of this species displayed only occasional loads of contaminants. Such low concentrations of contaminants are clearly in contrast to the marks of biofouling in long-term wetted surfaces such as ship hulls or underwater structures. In this way, our results may indicate a high quality of self-cleaning 
abilities of the skin of pilot whales (Globicephala melas), potentially in balance with the marks of biofouling reported previously (Gol'din 1994; Denys 1997; Baum et al. 2000).

In the present study we found evidence that self-cleaning relies on the patchy distribution of epidermal desquamation and a cellular morphology that was considered to be the basis of skin surface smoothness. Considering the presence of proteolytic enzymes within the intercellular space of the stratum corneum conjunctum in pilot whale (Baum et al. 2001b), the enzymatic digestion applied shows that serine proteases, such as trypsin, known in other mammals (e.g. Suzuki et al. 1996) may be responsible for the time-dependent process of desquamation and its patchy distribution. In the present study, the enzymatic digestion applied introduced a desquamation pattern not only in single squames $(30 \times 50 \mu \mathrm{m})$, but also in cell clusters (of up to $100 \times 300 \mu \mathrm{m}$ ). This pattern of desquamation, induced

in vitro in formol-fixed samples, reflected the patchy distribution of the mucilaginous, skin-covering fluid seen in some cryo-scanned fresh skin samples (Baum et al.2000). Although the efficiency of rate-dependent desquamation, contributing to the self-cleaning, versus rate-dependent adhesion of contaminants is a dynamic feature of the attachment-release process, it is assumed that the patchy distribution of desquamating cell assemblages is an adaptation to remove contaminants of the same size as that of these assemblages from the skin surface.

With respect to the cellular morphology and roughness of the skin - as the surface character of the hard cellular substrate on which contaminants and biofoulers attach - our results obtained from fresh-frozen samples showed that the skin surface of the pilot whale exhibited an even, smooth surface, formed by the tips of the nanoridges obscured by a medium. Because these nanoridges resisted the proteolysis applied and the glycoconjugates protect desmosomal material against proteolysis (Walsh and Chapman 1991), we conclude that the nanoridges originated from desmosomal-keratinaceous material. The nanoridges found were recovered in the freshly freeze-etched and aldehyde-fixed samples in this study. The morphology of these nanostructures was in agreement with previous reports on the skin of other delphinid species, in which the nanoridges were considered to be derivatives of intensely interdigitating desmosomal junctions (e.g. Pfeiffer and Jones 1993). Since the nanoridge-enclosed pores (diameter approximately 0.1 and $1.2 \mu \mathrm{m}^{2}$ ) measured in our study were smaller than most marine biofouling organisms (diameter $\leq 0.2 \mu \mathrm{m}$ ), we conclude that the biofoulers can attach to the skin surface only at

the margins of the pores or the tips of the nanoridges. Focusing on the reduction of available space for attachment and anchorage on the dolphin skin as a hard substratum, we claim that the plane of attachment of biofoulers is restricted to the level of the margins of the pores or the tips of the nanoridges. Biofoulers cannot invade deeper into the pores without heavy enzymatic (keratinolytic) digestion. Although further investigations are needed to evaluate the surface chemistry of the skin and the attachment dynamics of contaminants, this size exclusion is extremely important in the reduction of microniches (see below).

For biological surfaces exposed to air, such a reduction of effective, attachable contact space for contaminants has been reported for insects and plants (Wagner et al. 1996; Barthlott and Neinhuis 1997). In these organisms, microrough, waxy surface projections of about 1-10 $\mu \mathrm{m}$, with low wettability, absorb contaminants less effectively than water droplets which run over the surface and ultimately remove the contaminants. The viscoelastic and glycoprotein-rich thin mucoid layer covering the skin surface of fish prohibits direct contact of biofouling organisms with the body, whereby the mucus is retained in place by the microridges (approximately $10 \mu \mathrm{m}$ ) of the keratinocytes (e.g. Bereiter-Hahn et al. 1979; Mittal et al. 1995; Wirth 1999). In dolphins, the height of the nanoridges may correlate with the presence of an enzyme-containing gel, which fills the aforementioned structures with a medium of high elasticity. Thus, comparable skin-surface conditions may have evolved more than once early in the evolutionary development of fish and of those mammals resettling in aquatic environments.

Since the microrough projections described in insects and plants would - if present in dolphins - 
increase hydrodynamic drag, we assume that in dolphins the smooth plane (biofilm-filled nanorough surface system) evolved as an adaptation to the specific hydrodynamic demands of this mammalian group. As reported by Nachtigall (2000), small aquatic organisms survive in the laminar boundary of underwater surfaces, taking advantage of the microniches of their substrates. With respect to the nanoridges of the pilot whale forming an average nanorough plane surface, we established that the skin surface in dolphins exhibits no particular microniches for settling organisms and particulates

$(\leq 0.2 \mu \mathrm{m})$. This seems important, since the detachment forces in the laminar boundary layer are considered to be small (Schlichting and Gersten 1997; Nachtigall 2000), and contaminants penetrating into this water layer are not challenged by detachment forces.

From the results obtained with the above-reported experimental approach, we suggest a new model for the short-term self-cleaning abilities of dolphins based on the nanoroughness of their skin surface (Fig. 6). According to the hydrodynamically favorable low-shear, laminar drag-reducing flows around the dolphin's body, a load of small contaminants can be avoided, but also the laminar boundary layer is not destabilized by epidermal emergences reaching the boundary layer flows (Schlichting and Gersten 1997). Therefore, the self-cleaning abilities based on the specific and hydrodynamic nanorough surface structure in dolphins can be directly coupled with the hydrodynamic demands and swimming behavior of the dolphins, including forced motion, surfacing and especially jumping of whales.

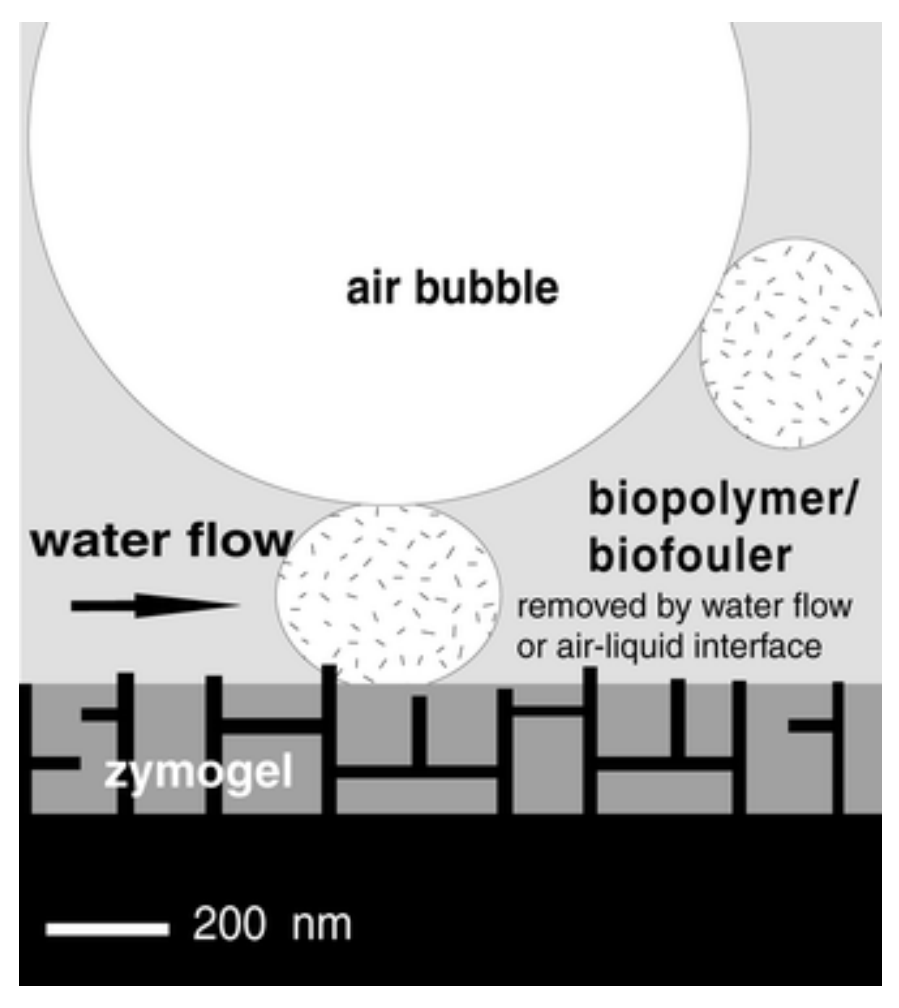

Fig. 6. Preliminary model of the self-cleaning abilities of pilot whale (Globicephala melas) skin, based on the relief of nanoridges filled with a medium. Larger biofoulers settling in the laminar boundary layer are excluded from the surface by the relief and can be flushed away by turbulent water flow or are removed by detachment forces of an air-liquid interface during breathing intervals or jumping of the dolphin. Smaller adhesive molecules are excluded from the surface by the properties of the gel

During jumping, attached particles not protected from air bubbles within microniches contact air-liquid interfaces or high-speed water flow, which reduce the height of laminar boundary layers; detaching forces are thus increased and the retention of such particles is considerably reduced. 
Acknowledgements. We thank Dr. D. Bloch, Museum of Natural History, Faroe Islands, Dr. H.-P. Joensen, University of the Faroe Islands, and co-workers for their kind help in specimen collecting from legal harvest. The authors also thank Dr. U. Passow and Dr. R. Crawford, Foundation Alfred-Wegener-Institute for Polar and Marine Research, for discussing the manuscript. This work was supported by a grant from the Deutsche Forschungsgemeinschaft DFG (ME 1755 1-1/1-2).

\section{References}

Aluwihare LI, Repeta DJ (1999) A comparison of the chemical characteristics of oceanic DOM and extracellular DOM produced by marine algae. Mar Ecol Prog Ser 186:105-117

Aluwihare LI, Repeta DJ, Chen RF (1997) A major biopolymeric component to dissolved organic carbon in surface sea water. Nature 387:166-169

Barthlott W, Neinhuis C (1997) Purity of the sacred lotus, or escape from contamination in biological surfaces. Planta 202:1-8

Baum C, Stelzer R, Meyer W, Fleischer L-G, Siebers D (2000) A cryo-scanning electron microscopic study of the skin surface of the pilot whale Globicephala melas. Aquat Mamm 26:7-16

Baum C, Fleischer L-G, Meyer W, Siebers D, Stelzer R (2001a) Gelation kinetics of the gel collected from the corneocytes of the pilot whale, Globicephala melas. Zoology (Jena) 104[Suppl IV]:53

Baum C, Meyer W, Roessner D, Fleischer L-G, Siebers D (2001b) A zymogel enhances the self-cleaning abilities of the pilot whale (Globicephala melas). Comp Biochem Physiol A 130:835-847

Bereiter-Hahn J, Osborn M, Weber K, Voeth M (1979) Filament organization and formation of microridges at the surface of fish epidermis. J Ultrastruct Res 69:316-330

Beveridge TJ, Makin SA, Kadurugamuva JL, Li Z (1997) Interactions between biofilm and the environment. FEMS Microbiol Rev 20:291-303

Chin W-C, Orellana MV, Verdugo P (1998) Spontaneous assembly of marine dissolved organic matter into polymer gels. Nature 391:568-572

Cooksey KE, Wigglesworth-Cooksey B (1995) Adhesion of bacteria and diatoms to surfaces in the sea: a review. Aquat Microb Ecol 9:87-96

Denys L (1997) Morphology and taxonomy of epizoic diatoms (Epiphalania and Tursiocola) on the sperm whale (Physeter macrocephalus) stranded on the coast of Belgium. Diatom Res 1:1-18

Dey S, Basu Baul TS, Roy B, Dey D (1989) A new arid method of air-drying for scanning microscopy using tetramethylsilane. J Microsc (Oxf) 156:259-261

Gol'din EB (1994) The Black Sea bottlenose dolphin (Tursiops truncatus) and overgrowing microalgae: same aspects of interrelations. In: Proceedings of the 1 st international symposium on the marine mammals of the Black Sea. Intergovernmental Oceanographic Commission, UNESCO, Istanbul, pp 24-27

Meyer W (1986) Die Haut des Schweines (The Porcine Integument). Vergleichende histologische und histochemische Untersuchungen an der Haut von Wildschweinen, Hausschweinen und Kleinschweinen. Schlütersche, Hannover 
Mittal AK, Garg TK, Verma M (1995) Surface architecture of the Indian catfish, Bagar bagarius (Hamilton) (Sisoridae; Siluriformes). Jpn J Ichthyol 42:187-191

Nachtigall W (2000) Leben in der Grenzschicht. Biol Unserer Zeit 30:148-157

Passow U (2000) Formation of transparent exopolymer particles, TEP, from dissolved precursor material. Mar Ecol Prog Ser 192:1-11

Pfeiffer CJ, Jones FM (1993) Epidermal lipid in several cetacean species: ultrastructural observations. Anat Embryol 188:209-218

Schlichting H, Gersten K (1997) Grenzschicht-Theorie, 9th edn. Springer, Heidelberg Berlin New York

Stoodley P, Lewandowski Z, Boyle JD, Lappin-Scott HM (1999) Structural deformation of bacterial biofilms caused by short-term fluctuations in fluid shear: an in situ investigation of biofilm rheology. Biotechnol Bioeng 65:83-92

Suzuki Y, Nomura J, Koyama J, Horii I (1996) The role of proteases in stratum corneum: involvement in stratum corneum desquamation. Arch Dermatol Res 286:249-253

Wagner T, Neinhuis C, Barthlott W (1996) Wettability and contaminability of insect wings as a function of their surface sculpture. Acta Zool 77:213-225

Wahl M (1989) Marine epibiosis. I. Fouling and antifouling: some basic aspects. Mar Ecol Prog Ser 58:175-189

Walsh A, Chapman SJ (1991) Sugars protect desmosome and corneosome glycoproteins from proteolysis. Arch Dermatol Res 283:174-179

Wirth U (1999) Vergleichende Untersuchungen zur Struktur und Funktion der Hautschichten bei Fischen unter besonderer Berücksichtigung von Hautsekreten. Dissertation thesis, University of Hannover 